\title{
Implementation of a nurse-led lower urinary tract symptoms (LUTS) clinic reduces general urology clinic workload in a Model 4 Hospital: a pilot study in Tallaght University Hospital
}

\author{
Kevin G. Keane ${ }^{1}$ (D) $\cdot$ Mohammud Shakeel Inder ${ }^{1} \cdot$ Caroline Mclntyre $^{1} \cdot$ Shawgi Omer $^{1} \cdot$ Elizabeth McEvoy $^{1}$. \\ Lisa G. Smyth ${ }^{1} \cdot$ Rowan G. Casey $^{1} \cdot$ Arun Z. Thomas $^{1,2} \cdot$ Rustom P. Manecksha ${ }^{1,2} \cdot$ Robert J. Flynn ${ }^{1,2}$
}

Received: 5 September 2020 / Accepted: 30 October 2020 / Published online: 14 November 2020

(C) Royal Academy of Medicine in Ireland 2020

\begin{abstract}
Background With among the lowest urologist per population ratios in Europe, the demand for urology specialist review in Ireland far exceeds supply. Lower urinary tract symptoms (LUTS) account for a significant number of referrals. The traditional paradigm of every patient being reviewed in a consultant-led clinic is unsustainable. New models of care with nurse-led clinics represent an opportunity to optimise limited resources.

Methods Existing long-waiting male LUTS referrals were triaged to a specialist nurse-led LUTS clinic. After urology CNS assessment, charts were reviewed by a consultant urologist and a plan formulated. Relevant data were prospectively collected and analysed.

Results Fifty-eight new male patients with LUTS were seen over a 6-month period with an average waiting time of 15.8 months. Patients were assessed with uroflowmetry, IPSS and DRE. Mean age was 64, IPSS 14.5, Qmax $18.3 \mathrm{ml} / \mathrm{s}$ and PVR $89 \mathrm{ml}$. Thirty patients $(52 \%)$ were discharged directly with lifestyle modification and medical therapy.

Twenty-eight patients (48\%) required one or more further investigations and subsequent review; 11 had flexible cystoscopy, 4 had urodynamics, 5 had prostate MRI, and 2 patients were listed for surgery (TURP and circumcision). The remaining 10 patients were for review post trial of lifestyle modifications and/or medical treatment. After review/investigations, 4 more patients were discharged. A total of 32 patients (55\%) were discharged or listed for surgery after initial assessment. This total increased to $62 \%$ after a second review/investigations.

Conclusion Introduction of a CNS-led LUTS clinic has significantly reduced the number of patients requiring follow-up in general urology clinics, representing a quality improvement in service provision.
\end{abstract}

Keywords Clinic $\cdot$ LUTS $\cdot$ Nurse-led $\cdot$ Urology

\section{Introduction}

Lower urinary tract symptoms (LUTS) is the term used to describe the constellation of symptoms experienced by patients relating to the bladder, prostate and urethra. LUTS account for a substantial number of referrals to the urology

Kevin G. Keane

kevin.keane@ucdconnect.ie

1 Department of Urology, Tallaght University Hospital, Dublin, Ireland

2 Department of Surgery, School of Medicine, Trinity College Dublin, Dublin, Ireland outpatient service and can be a cause of considerable distress to patients, effecting their physical, mental and social health [1]. Urology is a surgical speciality with the potential to significantly improve the quality of life of patients, often with minimal intervention. Currently, however, access to this care is lacking, with long delays from referral to consultation.

With among the lowest urologist per population ratios in Europe, the demand for urology specialist review in Ireland far exceeds supply [2]. The traditional paradigm of every patient being reviewed in a consultant-led clinic is unsustainable. A new model of care for urology in Ireland proposing radical changes to the way care is delivered was released in 2019 [3]. The report outlines a shift of care towards the community with more of an emphasis on the multidisciplinary role of specialist nurses, allied health professionals and general practitioners. 
The new model of care contains suggestions to improve the provision of urological services in Ireland including in the development of dedicated referral pathways with particular mention given to the implementation of a nurse-led LUTS clinic within each hospital group.

Following on from a successful pilot implementation in a Model 3 hospital in Letterkenny, we hypothesised that establishing a nurse-led LUTS clinic in a Model 4 hospital would significantly reduce waiting times for patients, improve access to urological care and reduce the overwhelming burden currently experienced in general urology outpatient clinics [4]. The aim of our pilot study was to objectively demonstrate that urology nurse specialists can safely run a benign urology clinic in well-prepared settings.

\section{Methods}

This was a prospective observational cohort study carried out in Tallaght University Hospital over a 6-month period between September 2019 and February 2020. All referrals to the urology service were screened by a consultant urologist. Existing suitable long-waiting male LUTS referrals were triaged directly to a newly established LUTS clinic run by a single Urology Clinical Nurse Specialist (CNS). Exclusion criteria included females with LUTS, patients who had already been reviewed in general urology clinic, patients who had previously undergone surgery for LUTS and those with an elevated PSA.

Patients were clearly informed that they would be reviewed by a Urology CNS and not a urologist. A designated clinic room in the urology outpatient suite was used for all appointments and contained a patient examination table, toilet, urine flow metre and bladder scanner, with access to urinalysis and blood testing.

Patients completed an International Prostate Symptom Score (IPSS) in the waiting room. The IPSS is a validated scoring tool which objectively assesses the severity of LUTS and can be used to monitor response to treatment. Subsequently, a thorough medical and focused urological history was taken by the urology CNS. All medications were scrutinised and recorded. All patients underwent general examination as well as digital rectal examination (DRE) performed by the urology CNS unless declined. Following this, patients went for uroflowmetry after which a post void residual (PVR) was recorded. If necessary, patients were sent for blood tests and urinalysis.

Patients were counselled on the potential aetiology of their LUTS and provided with an interpretation of their results. All patients were educated on the role dietary and lifestyle modifications may have on controlling their
LUTS. Patients were then informed of their management plan and given the opportunity to ask questions at the end of the consultation (Fig. 1).

After CNS assessment, the patient's charts were reviewed by a consultant urologist and a plan formulated. At this point, relevant medications such as alpha-blockers, 5-alpha reductase inhibitors or antimuscarinics were prescribed. Patients were triaged to return to nurse-led LUTS clinic after an interval, be discharged back to the GP for follow-up in the community or come to a general urology consultant-led clinic depending on the scenario.

Data was collected prospectively and stored on a secure folder on the hospital server. Information collected included basic demographics, length of time on the waiting list, significant past medical history, baseline medications, IPSS, DRE findings, uroflowmetry results (Qmax, Qavg), post void residual, PSA, urinalysis findings, bloods tests or imaging to date, management plan and clinical outcome (Fig. 2). Statistical analysis was done in SPSS V.25 (IBM Corp. Released 2017. IBM SPSS Statistics for Windows, Version 25.0. Armonk, NY: IBM Corp.).

\section{Results}

\section{Baseline characteristics}

A total of 58 new male patients were assessed for LUTS over a 6 -month period. The average waiting time from referral to review was 63.2 weeks $(\mathrm{SD} \pm 20)$. The mean age of patients was $64(\mathrm{SD} \pm 12.5)$. All patients completed an IPSS, with a mean score of 14.5 ( $\mathrm{SD} \pm 6.8)$ (Table 1). The IPSS was classified as moderate or severe in $82 \%$ of patients $(57 \%$ moderate, $25 \%$ severe) and mild in $18 \%$ of patients.

\section{Investigations}

DRE was performed in all but 4 patients and documented as suspicious in 5 patients. Uroflowmetry was performed in all patients. All patients voided $>150 \mathrm{ml}$ urine allowing for diagnostic interpretation. The mean maximum flow rate (Qmax) was $18.3 \mathrm{ml} / \mathrm{s}(\mathrm{SD} \pm 8.3)$, and $37 \%$ of patients $(n=22)$ had a Qmax of $<15 \mathrm{ml} / \mathrm{s}$. The average post void residual (PVR) was $89 \mathrm{ml}(\mathrm{SD} \pm 127)$ (Table 1).

\section{Outcomes}

Thirty patients $(52 \%)$ were discharged directly back to the GP with lifestyle modification \pm medical therapy following initial assessment by the urology CNS and review of chart by a consultant urologist. Alpha-blockers were prescribed to $27 \%$ of patients $(n=16)$ and anticholinergics to $15 \%$ of patients $(n=9)$, and 1 patient was commenced on 
Fig. 1 Review process

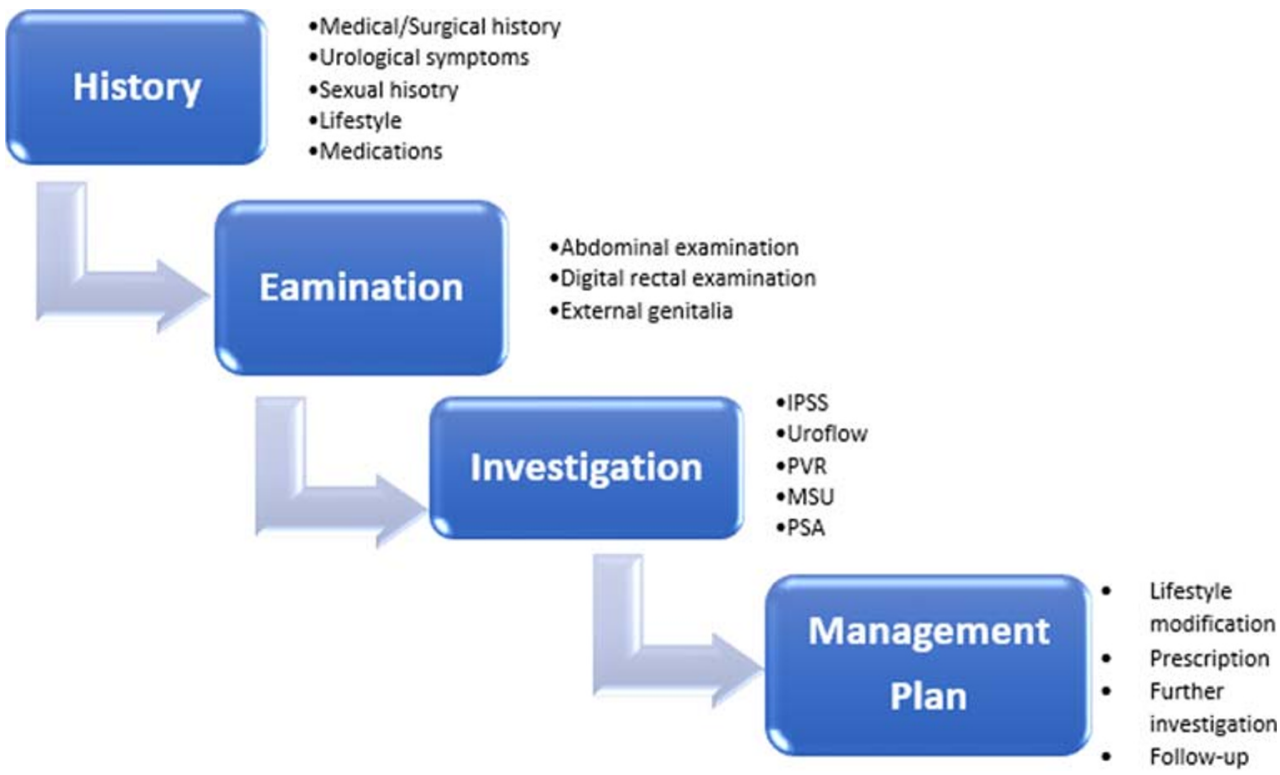

a 5 alpha-reductase inhibitor. Twenty-eight patients (48\%) required further review or investigations after initial assessment; 11 had flexible cystoscopy, 4 had urodynamic studies, 5 had prostate MRI, and 2 patients were listed for surgery following discussion with a consultant (TURP and circumcision). The remaining 10 patients were for review post trial of lifestyle modifications and/or medical treatment. After review/investigations, 4 more patients were discharged (Fig. 3).

A total of 32 patients $(55 \%)$ were discharged or listed for surgery after initial assessment. This total increased to $62 \%$ after a second review/investigations.

\section{Discussion}

Urology is the 4th busiest surgical speciality in Ireland based on discharges [3]. Ireland's population is living longer, and this has directly impacted the number of patients with urological symptoms. In 2019, there were 30,905 patients waiting to see a urologist, and this number is estimated to increase to 46,729 patients by 2022 [3]. There is a significant bottleneck when it comes to accessing a urologist in the public sector leading to long delays in care. Patients with LUTS tend to be of low priority when triaged in favour of more urgent cases such as cancer.
Fig. 2 LUTS assessment pro forma

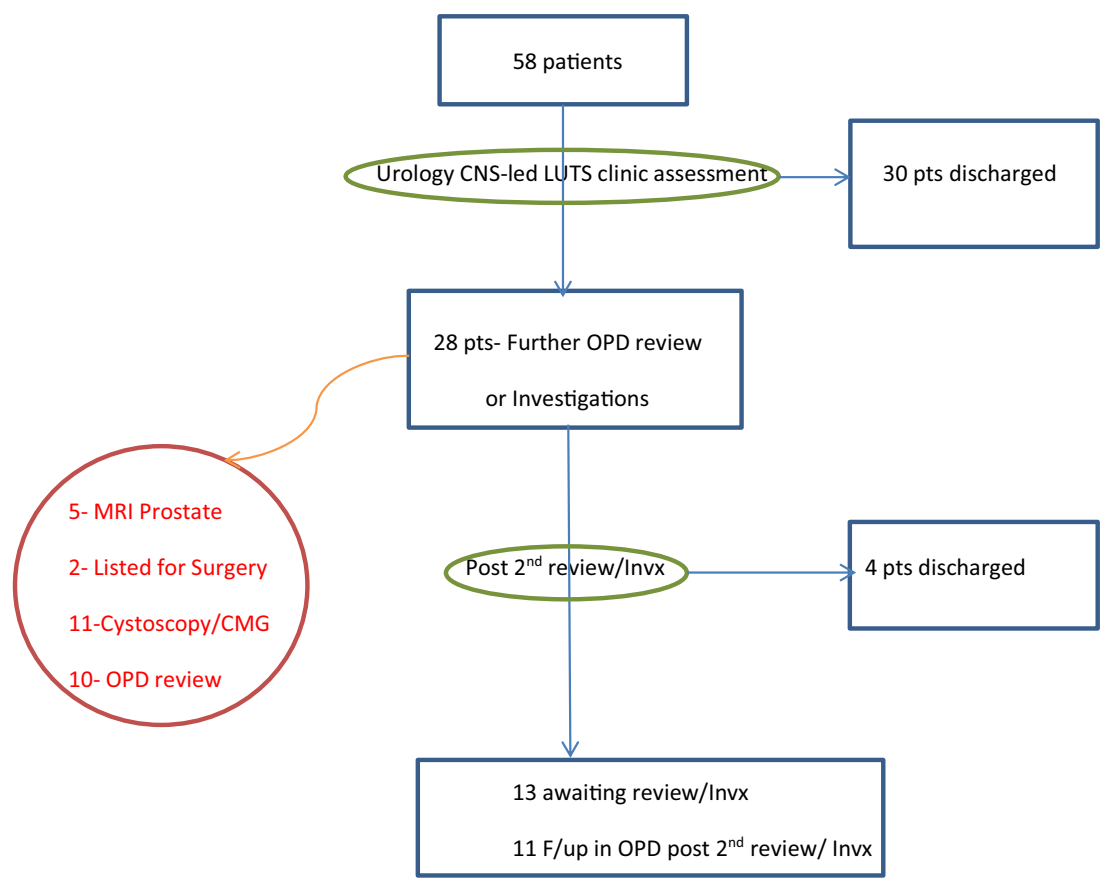


Table 1 Characteristics and investigations

$\begin{array}{ll}\text { Mean age (years) } & 64(\mathrm{SD} \pm 12.5) \\ \text { Mean time from referral to consultation (weeks) } & 63.2(\mathrm{SD} \pm 20) \\ \text { International Prostate Symptom Score } & N=11 \\ \text { Mild (1-7) } & N=32 \\ \text { Moderate (8-19) } & N=15 \\ \text { Severe (20-35) } & 18.3(\mathrm{SD} \pm 8.3) \\ \text { Mean Qmax flow rate }(\mathrm{ml} / \mathrm{s}) & 8.8(\mathrm{SD} \pm 4.4) \\ \text { Mean Qavg flow rate }(\mathrm{ml} / \mathrm{s}) & 89(\mathrm{SD} \pm 127) \\ \text { Mean post void residual }(\mathrm{ml}) & 1.8(\mathrm{SD} \pm 10.32) \\ \text { Mean PSA (mg/ml) } & \end{array}$

The nurse-led LUTS clinic is not designed to replace general urology outpatient clinics but to greatly reduce the number of patients requiring review by a urologist [5]. Our aim was to objectively demonstrate that urology nurse specialists can safely run a benign urology clinic in well-prepared settings. We report an initial direct discharge back to community care rate of $52 \%$ following pilot implementation of a nurse-led male LUTS clinic in a Model 4 hospital. Furthermore, those patients identified in the LUTS clinic as requiring urologist review were sent for the appropriate investigations before being seen, allowing the urologist to make informed decisions about their care and construct an adequate management plan without further delay.

Urology has evolved rapidly as a specialty over the last 50 years. The management of LUTS has moved towards diagnostic tests followed by medications, with surgery generally reserved for failure of medical management. A substantial amount of low complexity LUTS can be managed effectively with re-assurance and increased awareness of the role medications, lifestyle and dietary factors play on symptoms. Educational interventions such as double voiding technique, the use of bladder diaries, avoidance of constipation and fluid management can be performed successfully by urology nurses and GPs with a special interest in urology. Loftus et al. reported a positive impact on the quality of outpatient care for patients when delivered by specialist nurses, and a recent Irish audit highlighted the success of nurse-led continence clinics, citing a $66 \%$ improvement in symptoms on discharge $[3,6]$. In our experience, patients seemed pleased by a more holistic approach to their care and longer consultation time with the urology CNS than they would have experienced in a doctors clinic.

The prioritisation of referrals framework presented in the model for care recommends that patients with severe LUTS, as characterised by the IPSS, be seen within 28 days and those with moderate LUTS within 26 weeks[3]. In our study, 55\% of patients were referred with moderate LUTS and $25 \%$ with severe LUTS. The mean waiting time to be seen was 63 weeks. This is much longer than new recommendations and is a representation of the overstretched healthcare system which we currently operate in. In our study, no patient was referred with a completed IPSS. In order to adhere to the model of care prioritisation strategy and accurately triage patients based on urgency, greater buy in is needed at a community level. All male patients with LUTS should have an IPSS and DRE performed by the GP prior to urology referral (Fig. 4).

An ageing demographic is not solely to blame for the long waiting times to access urology services in Ireland. A report by the American Urological Association in 1995 cited 1

Fig. 3 Patient flow
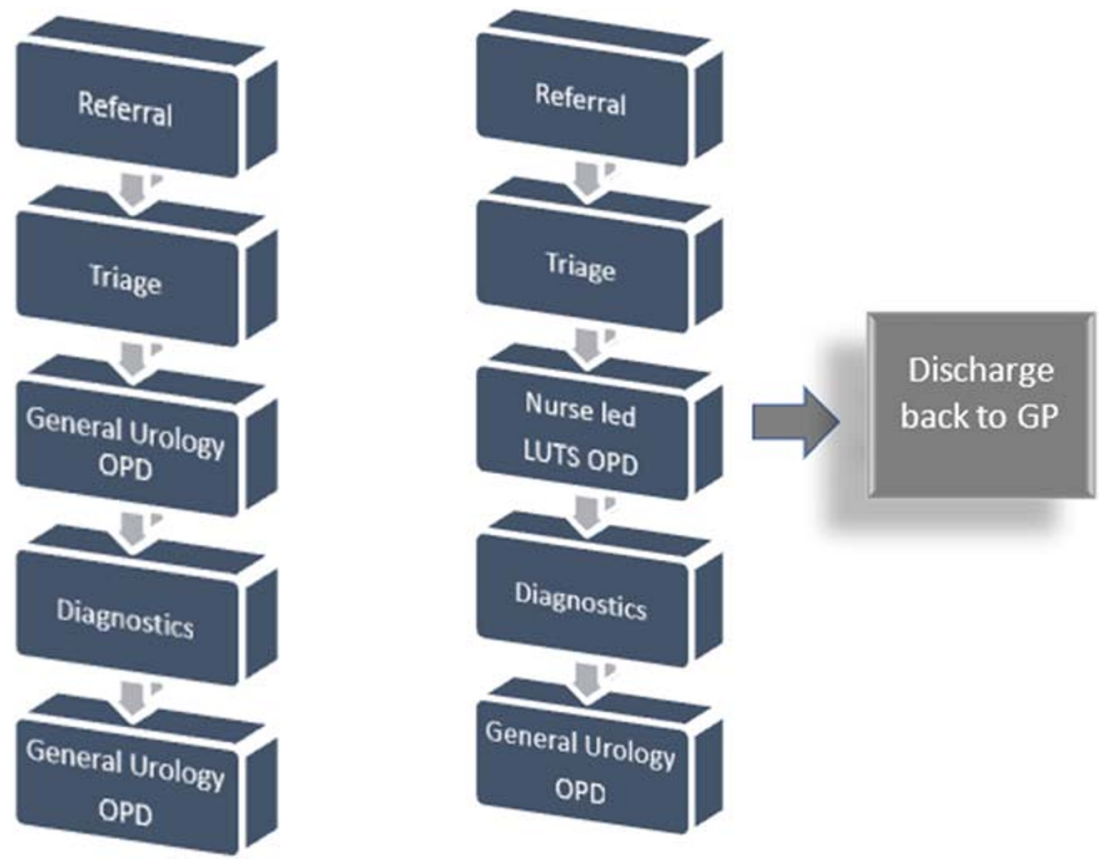


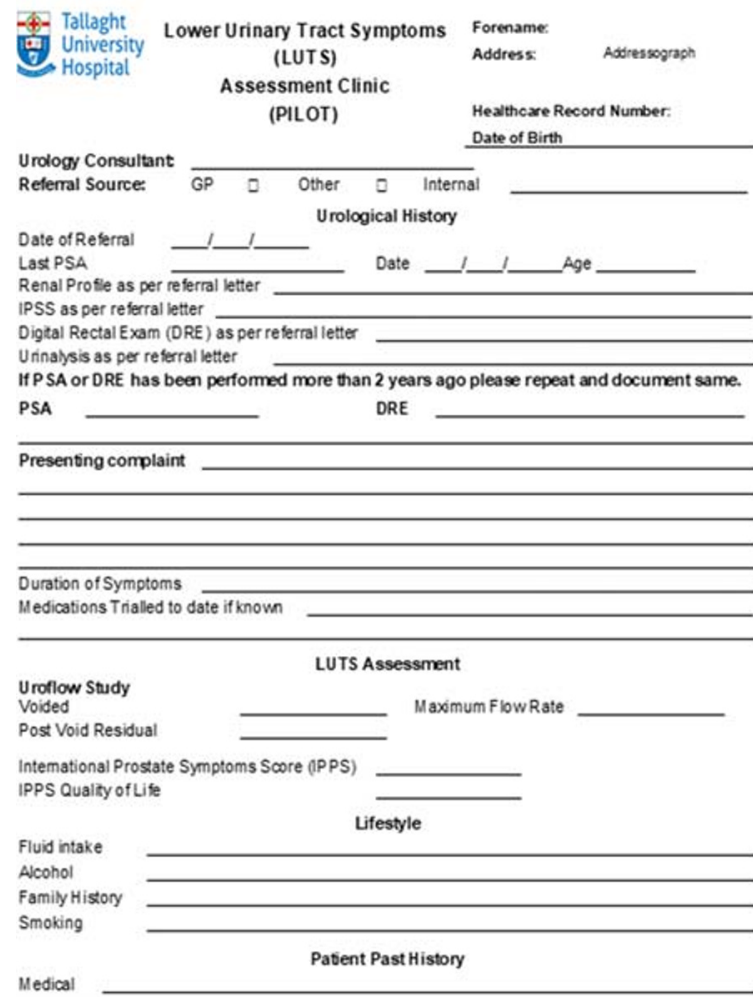

Fig. 4 Traditional vs nurse-led LUTS clinic paradigm

urologist per 50,000 population as desirable to provide an effective service [7]. In 2016, the British Association of Urological Surgeons (BAUS) released a report showing Ireland had 1 urologist to 127,027 people, one of the lowest rates in the first world and half that of the UK [2]. A survey of Irish urology consultants in 2016 identified the development of nurse-led urology clinics as a priority of outpatient care [3]. The most prevalent symptoms referred to urology outpatient departments in Ireland were evaluated in 2017. There were 1000 patients waiting to be seen in Model 3 hospitals, with LUTS accounting for the second most common indication for referral after haematuria. When Model 4 hospitals were examined, after exclusion of patients referred with signs of prostate cancer, haematuria and LUTS again accounted for the vast majority of referrals [3]. Thus, it can be extrapolated that interventions aimed at targeting these patient cohorts would have the greatest impact on improving the efficiency of outpatient delivery of urological care.

The obvious limitation of our study is the small sample size. Unfortunately, due to the COVID-19 pandemic, the outpatient clinics at our institution were curtailed which led to the study being concluded prematurely. There is the potential for selection bias at the triage stage, and some patients may have been thought not suitable for a nurse-led clinic without meeting the exclusion criteria. Longer term follow-up of patients with objective measures such as a repeat IPSS score is warranted to conclusively determine the efficacy of a nurse-led

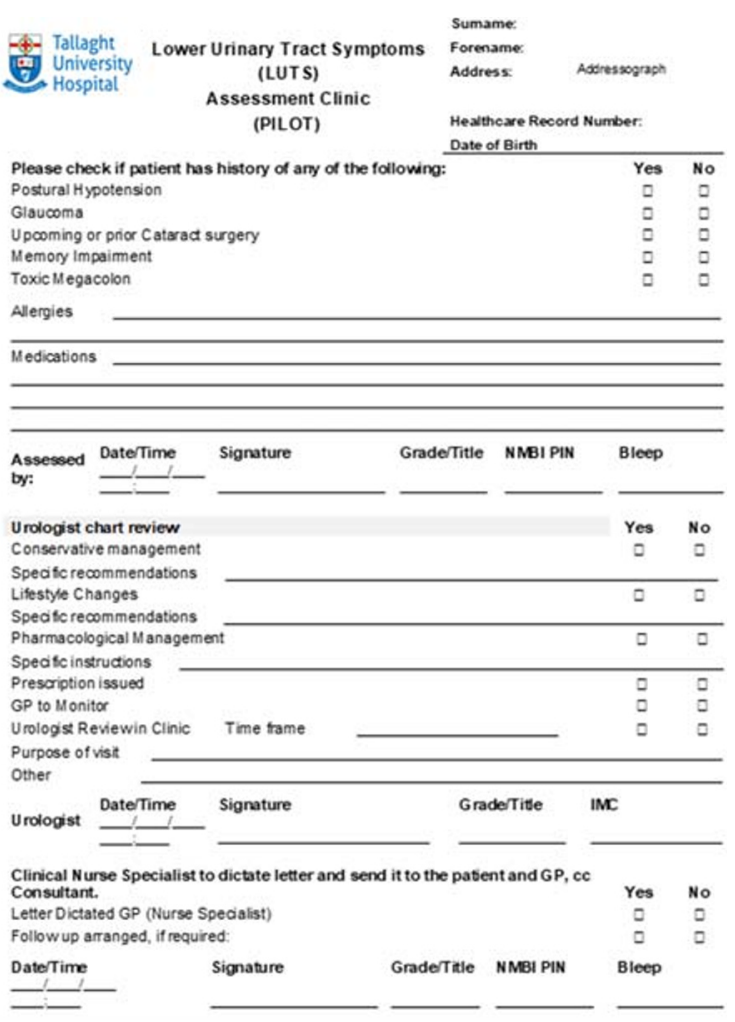

LUTS clinic. Despite this, our pilot study has suggested the feasibility of implementing a nurse-led LUTS clinic in Model 4 hospitals in Ireland, with the potential for a meaningful reduction in wait times for patients and workload for urologists.

Following on from the success of our pilot implementation of a CNS-led LUTS clinic, we have now established an Advanced Nurse Practitioner-led LUTS clinic. Advanced nurse practitioners can prescribe medications and order investigations directly. This will further reduce footfall through general urology clinic, shorten waiting time for patients and free urology trainees up to focus on surgical training.

\section{Conclusion}

Introduction of a nurse-led LUTS clinic has significantly improved waiting times for patients referred to Tallaght University Hospital and reduced the number of patients needing consultant urologist review. This pilot study supports the implementation of nurse-led LUTS clinics in Model 4 hospitals in Ireland.

Supplementary Information The online version contains supplementary material available at https://doi.org/10.1007/s11845-020-02428-8.

Data availability Yes 


\section{Compliance with ethical standards}

Conflicts of interest The authors declare they have no conflicts of interest

Ethics approval No intervention performed. Ethical approval waived

Consent for publication All authors consent to publication

\section{References}

1. Bruskewitz RC (2003) Quality of life and sexual function in patients with benign prostatic hyperplasia. Reviews in urology 5(2):72-80

2. Surgeons BAoU (2016) British Asociation of urological surgeons and the specialist advisory committee in urology workforce report. https://www.baus.org.uk/_userfiles/pages/files/About/Governance/ 2016\%20FINAL\%20Workforce\%20Report.pdf

3. RCSI/NCPS/HSE (2019) Urology; A model of care for Ireland. https://www.rcsi.com/surgery/-/media/feature/media/download- document/surgery/practice/publications-and-guidelines/models-ofcare/urology-a-modelof-care-for-ireland.pdf

4. Lonergan PE, Logan J, Diver S et al (2020) Does clinical validation and the implementation of new models of outpatient service delivery have the potential to reduce waiting lists? A pilot study in Letterkenny University Hospital. Ir J Med Sci 189(3):777-782. https://doi.org/10.1007/s11845-020-02183-w

5. Koo V, McMahon J, O’Brien A et al (2008) Outcome audit of nurseled lower urinary tract symptoms clinic: lessons and challenges for practice. Int J Urol Nurs 2(2):72-77. https://doi.org/10.1111/j.1749771X.2008.00057.x

6. Loftus LA, Weston V (2001) The development of nurse-led clinics in cancer care. J Clin Nurs 10(2):215-220. https://doi.org/10.1046/j. 1365-2702.2001.00488.x

7. Weiner M, McDaniel R, Lowe FC (1997) Urologic manpower issues for the 21st century: Assessing the impact of changing population demographics. Urology 49(3):335-342

Publisher's note Springer Nature remains neutral with regard to jurisdictional claims in published maps and institutional affiliations. 